\title{
Classification and diagnostic criteria for psoriatic arthritis
}

\section{P S Helliwell, W J Taylor}

Ann Rheum Dis 2005;64(Suppl II):ii3-ii8. doi: 10.1136/ard.2004.032318

\begin{abstract}
Background: The study of psoriatic arthritis is difficult and has lagged behind the study of other arthropathies in that there are no universally agreed or properly validated case definitions.

Method: This paper examined the validity and practicality of the original Moll and Wright criteria and subsequent criteria sets. Key features discriminating between psoriatic and other arthropathies were reviewed. A comparative study involving patients with psoriatic arthritis and rheumatoid arthritis was used to contrast the different classification methods.

Results: Although the Moll and Wright criteria continue to be widely used, they have been shown to discriminate poorly between psoriatic and rheumatoid arthritis. In comparison, the most sensitive criteria were those of Vasey and Espinoza, McGonagle et al, and Gladman et al $(99 \%)$, whereas the others were significantly less sensitive (between $56 \%$ and $94 \%)$. The specificity of all methods was high and statistically similar (between 93\% and 99\%). Models that had reasonably good accuracy even without such key variables as psoriasis or rheumatoid factor were developed. Spinal involvement continues to be a key feature of psoriatic arthritis, but dissimilarities with classic ankylosing spondylitis have been highlighted.
\end{abstract}

Conclusions: Further work is required to produce classification criteria for psoriatic arthritis. A prospective study collecting clinical, radiological, human leucocyte antigen (HLA) and immunological data from both psoriatic and nonpsoriatic cases should provide agreed criteria for use in psoriatic arthritis studies in the future.

U ntil the pioneering work of Wright $^{1}$ and Baker, inflammatory arthritis occurring in the presence of psoriasis was felt to represent rheumatoid arthritis (RA) occurring coincidentally with psoriasis. Wright described the frequent involvement of distal interphalangeal (DIP) joints with erosion and absorption of the terminal phalanges, coexisting sacroiliitis, involvement of the proximal interphalangeal (PIP) joints of the toes, and a characteristic mutilating arthritis with reduction in bone stock particularly in the digits. ${ }^{3}$ The discovery of rheumatoid factor in the serum helped categorise polyarthritis, but the distinction between RA and psoriatic arthritis (PsA) was and currently is defined on clinical grounds. The American College of Rheumatology (previously the American Rheumatism Association) adopted PsA as a distinct clinical entity, including it in a classification of rheumatic diseases for the first time in $1964 .^{5}$

Further studies culminated in the concept of the seronegative spondyloarthropathies as a group of disorders sharing common clinical features, including sacroiliitis, seronegative anodular asymmetrical peripheral oligoarthritis, hyperkeratotic and sometimes pustular rash on the hands and soles (keratoderma blennorrhagica), peripheral and central enthesitis, iridocyclitis, mucocutaneous ulceration, and familial aggregation. ${ }^{6}$ The discovery of the high prevalence of human leucocyte antigen (HLA)-B27 in ankylosing spondylitis and other diseases in this group provided confirmation of this concept. ${ }^{7}$

Despite clinical, radiological, and familial evidence supporting PsA as a distinct disease entity, controversy still exists about which patients to include within this disease group. Some authors have even questioned whether PsA is a separate disease, suggesting that psoriasis merely modifies the expression of pre-existing RA. ${ }^{8}$ Other authors have argued that new onset chronic polyarthritis is undifferentiated and only evolves into a more distinctive form with time such that the presence of psoriasis at onset of disease is of no value in nosological terms. ${ }^{9}$

Validated classification criteria have been developed for a number of rheumatic diseases. Such criteria are important for several reasons. They enable the classification of homogeneous groups to facilitate comparison between centres and different countries in the areas of epidemiology, outcome studies, and therapeutic trials. Agreed and validated criteria are critical to meaningful research into immunogenetics and other basic sciences. The most recent criteria for RA were derived from a sample of 262 RA cases using an equal number of other non-rheumatoid inflammatory arthritis cases as controls. ${ }^{10}$ The case selection was made by the physician on clinical grounds, in the absence of an appropriate biological marker. The concept involved agreeing on a set of clinical and other variables that were assessed in all cases. Case definitions were derived using univariate statistics followed by statistical techniques employing Boolean algebra to develop combinations of variables that produced maximal sensitivity and specificity for that data set. For RA, the disease definition listed seven criteria, patients needing to fulfil four of these to be included. Sensitivity was $91 \%$, specificity $89 \%$. A similar methodology was employed in the development of the European Spondyloarthropathy Study Group (ESSG) criteria for spondyloarthropathy (covering ankylosing spondylitis, reactive arthritis, the arthritis associated with inflammatory bowel disease, PsA and, importantly, an undifferentiated category). However, there was a notable difference in the data analysis; the authors specified the deliberate inclusion of at least one variable combining the specific variables of individual disease subgroups, even if these were not selected by multivariate analysis. ${ }^{11}$ The authors argued that this was necessary as the cases were dominated by one disease group: ankylosing spondylitis (42\%). Again, sensitivity (87\%) and specificity (87\%) were both acceptable. Although these and other criteria are widely used for their intended purpose, they continue to be used in the clinic, inappropriately, as diagnostic criteria for the individual patient. Classification studies are potentially

Abbreviations: CCP, cyclic citrullinated peptide; DIP, distal interphalangeal (joint); ESSG, European Spondyloarthropathy Study Group; HLA, human leucocyte antigen; PIP, proximal interphalangeal (joint); PsA, psoriatic arthritis; RA, rheumatoid arthritis 
subject to a number of important biases. These include circularity of criteria items (the same rheumatologists who judge whether the patient has the disease or not also develop the list of potential diagnostic items), selection bias (only cases or controls that are "classic" examples are included), and heterogeneity of case ascertainment (rheumatologists may not be diagnosing cases in the same way). Absence of a "gold standard" for case ascertainment underlies much of the difficulty.

Unfortunately, validated criteria such as those developed for RA do not yet exist for PsA. The development of new therapies, particularly biological therapies, has highlighted this deficiency and made the need for such criteria and for standardised outcome and response criteria more urgent. ${ }^{12}$ The situation is made more complex in PsA by the existence of several disease subgroups, the precise composition and relative proportions of which has been the subject of some debate. The problem is not with the classic presentation of PsA-that is, with oligoarthritis, DIP involvement, calcaneal enthesitis, and dactylitis-but with the group of patients who have seronegative polyarthritis and psoriasis.

In this paper, we review existing diagnostic classification criteria for PsA and some of the clinical features that may cause confusion and even overlap between PsA and other arthropathies, such as RA and ankylosing spondylitis.

\section{MOLL AND WRIGHT CRITERIA AND SPECIFIC FEATURES OF PsA \\ Moll and Wright criteria}

The original diagnostic criteria of Moll and Wright ${ }^{13}$ are the simplest and the most frequently used in current studies. The criteria are:

- an inflammatory arthritis (peripheral arthritis and/or sacroiliitis or spondylitis)

- the presence of psoriasis

- the (usual) absence of serological tests for rheumatoid factor.

Using these diagnostic criteria Moll and Wright described five subgroups of PsA: DIP joint only, asymmetrical oligoarthritis, polyarthritis, spondylitis, and arthritis mutilans.

Minor modifications have been made to the Moll and Wright subgroups by a number of authors including Gladman et al, ${ }^{14}$ Oriente et al, ${ }^{15}$ Helliwell et al, ${ }^{16}$ Torre-Alonso et $a l,{ }^{17}$ Jones et al, ${ }^{18}$ and Veale et al. ${ }^{19}$ It is of interest to compare the proportion of patients included in the polyarthritis and asymmetrical oligoarthritis subgroups by these authors (fig 1). To enable a direct comparison, patients described as belonging to more than one subgroup (spondylitis and one other of the peripheral subgroups) are classified into the peripheral subgroup as Moll and Wright also placed patients into their predominant subgroup for the purposes of their classification. Marked differences are seen between the original Moll and Wright series and the subsequent reports. Assuming that there have been no fundamental changes to the disease in the past 40 years, it must be concluded that subsequent authors have interpreted the diagnostic criteria and/or the subgroup criteria of Moll and Wright in different ways. The original criteria were designed to be sensitive without being too specific, but it is likely that Moll and Wright were using other features of the disease to make their diagnosis ( $\mathrm{V}$ Wright, personal communication). In other words, they were using implicit, but undeclared, features to enhance the specificity of their criteria. Later authors, unaware of this, have interpreted the Moll and Wright criteria meticulously-resulting in the inclusion of a higher percentage of patients with seronegative symmetrical

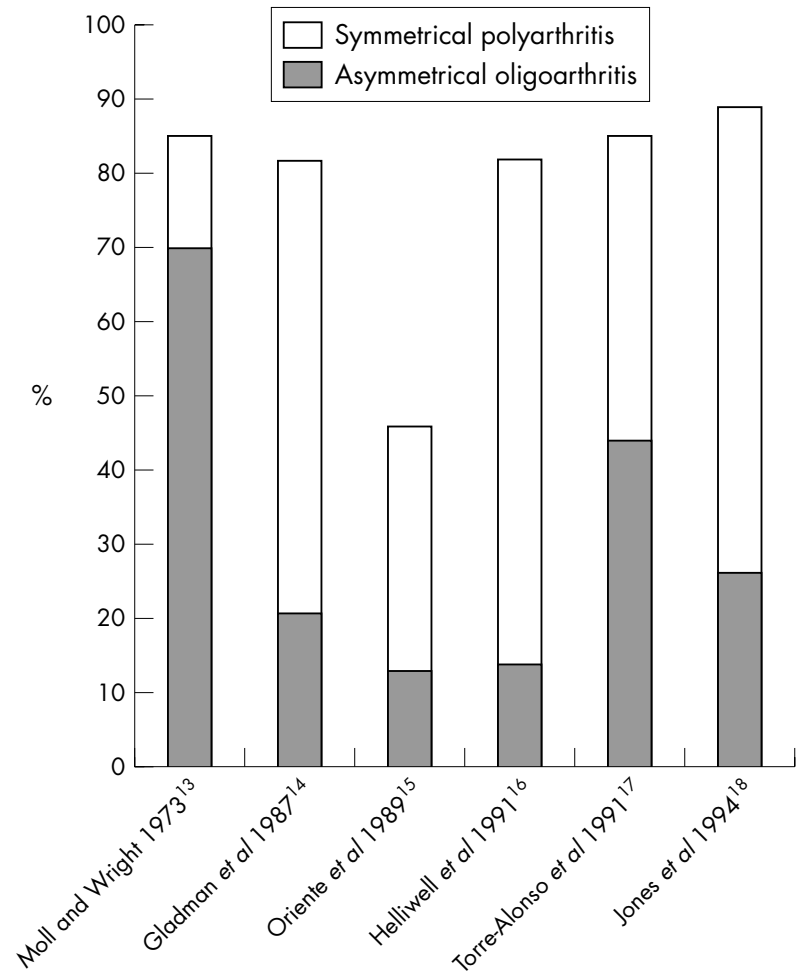

Figure 1 The proportion of patients in the asymmetrical oligoarthritis and symmetrical polyarthritis subgroups in several series of patients with psoriatic arthritis.

polyarthritis. As a result of this, it is possible that some of the patients included in the later series have seronegative RA with coincidental psoriasis. In addition, some of the criteria emphasised by Moll and Wright do not endure further scrutiny, and these will be briefly discussed.

\section{Rheumatoid factor}

The unwitting inclusion of patients with RA may be especially true if patients positive for rheumatoid factor are included in the series. Gladman et al have argued that there is no reason to insist on seronegativity for rheumatoid factor as this factor is found to be positive in people unaffected by arthritis, particularly if they have a chronic inflammatory disorder such as psoriasis. ${ }^{14}$ In fact, $12 \%$ of cases in Gladman's series were seropositive for rheumatoid factor, but the authors were careful to exclude patients who displayed other characteristic signs of RA such as nodules or extra-articular involvement. However, the inclusion of patients who are seropositive for rheumatoid factor does not explain the differences observed in the other series in which these patients were excluded.

It may also be possible to enhance the specificity of rheumatoid factor by identifying its different isotypes, IgA and IgG rheumatoid factor having greater specificity for RA. ${ }^{20}$ It may also be possible to separate seronegative RA from PsA by the use of other more specific autoantibody assays, particularly antifilaggrin antibodies. One such antibody, anticyclic citrullinated peptide (anti-CCP), has shown much higher specificity for RA than IgM RF. ${ }^{21}$

\section{Joint symmetry}

One of the clinical characteristics not explicit in the Moll and Wright criteria, but nevertheless emphasised in the accompanying papers, was the asymmetry of the disease, particularly in the oligoarthritis subgroup. ${ }^{13}$ However, a definition of 
symmetry was not provided. Further work from the Leeds group used stricter criteria ${ }^{16}$ and subsequently demonstrated that symmetry is not an inherent and distinctive feature of PsA but a function of the number of joints involved. ${ }^{22}$ The fact that patients with early and late PsA have fewer joints involved clinically accounts for the asymmetry of this disease. In fact this may only be a relative asymmetry as clinical examination is a relatively insensitive way of identifying articular involvement; the use of other modalities to identify involvement, such as colour Doppler ultrasound and magnetic resonance imaging (MRI) may demonstrate that articular and entheseal involvement in these arthritides is a quantitative rather than a qualitative distinction.

\section{The presence of psoriasis}

A further problem with distinguishing PsA from other arthropathies, in particular RA, is the almost universal mandatory criterion of the presence of psoriasis. The pitfalls associated with this are summarised below.

- Psoriasis is a common skin disease occurring in about $2 \%$ of the north European population..$^{23}$ Although HLA studies suggest different associations with psoriasis and RA, the diseases are not mutually exclusive, and some cases of RA will have coincidental psoriasis by chance alone.

- Psoriasis may precede, occur simultaneously, or follow the onset of arthritis. ${ }^{24}$ In the latter case, the patient may be mistakenly diagnosed as having an inflammatory arthritis other than PsA. In this situation, a family history of psoriasis may be an important clue as indicated below.

- Psoriasis may be present but may be hidden or may be misdiagnosed (by rheumatologists). ${ }^{25}$ Psoriasis may only be apparent in the natal cleft or some other "hidden" area such as under the breasts, around the umbilicus, or in the hairline. The psoriasis may only be evident in the nails.

- In the true absence of psoriasis, a positive family history in a first degree relative may be of equal importance from a diagnostic point of view. ${ }^{26}$

\section{Spinal involvement}

In addition to peripheral arthritis, people with psoriasis are also more likely to develop an inflammatory spinal disease similar to ankylosing spondylitis. The inflammatory spinal disease may be indistinguishable from ankylosing spondylitis but may differ from the classic disease in several respects. These differences were originally described by McEwen and colleagues $^{27}$ and, in part, later confirmed by Helliwell et al. ${ }^{28}$
The features more often seen in association with psoriasis (and reactive arthritis) can be summarised as follows:

- asymmetrical sacroiliitis

- non-marginal syndesmophytes

- asymmetrical syndesmophytes

- paravertebral ossification

- more frequent involvement of cervical spine.

In fact, the later study by Helliwell et al found paravertebral ossification to be so rare as to be of little value in discrimination, and the predominance of cervical spine involvement was a result of a relative sparing of the lumbar spine in psoriatic spondylitis. Further work by de Vlam et al has suggested a possible mechanism for the non-marginal "bulky" syndesmophytes, which are seen more frequently in association with psoriasis. ${ }^{29}$ de Vlam argued that syndesmophyte morphology is simply a result of the amount of mobility in the adjacent spinal segment; if the segment continues to have mobility in the zygoapophyseal joints, then the syndesmophyte is more likely to be "chunky". de Vlam demonstrated an association between zygoapophyseal fusion and classic marginal syndesmophytes, finding chunky syndesmophytes where posterior fusion had not occurred.

Can the differences between psoriatic and classic ankylosing spondylitis be, as in the case of symmetry, ascribed to quantitative rather than qualitative differences? There would certainly be some support for this, particularly if the difference in syndesmophyte morphology can be explained by the co-existing involvement of the zygoapophyseal joints. One further point is of interest with respect to spinal involvement, which may be of importance in relation to diagnosis and classification: the frequent asymptomatic involvement of the spine and sacroiliac joints in association with psoriasis. ${ }^{30}$ Clearly, diagnosis and classification on symptoms alone are insufficient and some form of objective imaging appears necessary to complete the picture.

Moll and Wright played a major part in the early work on the diagnosis and classification of PsA. However, their existing criteria need modification to make them more specific. Individual criteria, such as the presence of psoriasis and the absence of rheumatoid factor require further evaluation in these modified criteria sets.

\section{OTHER DIAGNOSTIC CRITERIA SETS}

Mention has already been made of the work of Gladman and colleagues who sought to modify the Moll and Wright criteria by adding a list of exclusion criteria to those proposed by

Table 1 Criteria for psoriatic arthritis (PsA) proposed by Bennett ${ }^{31}$

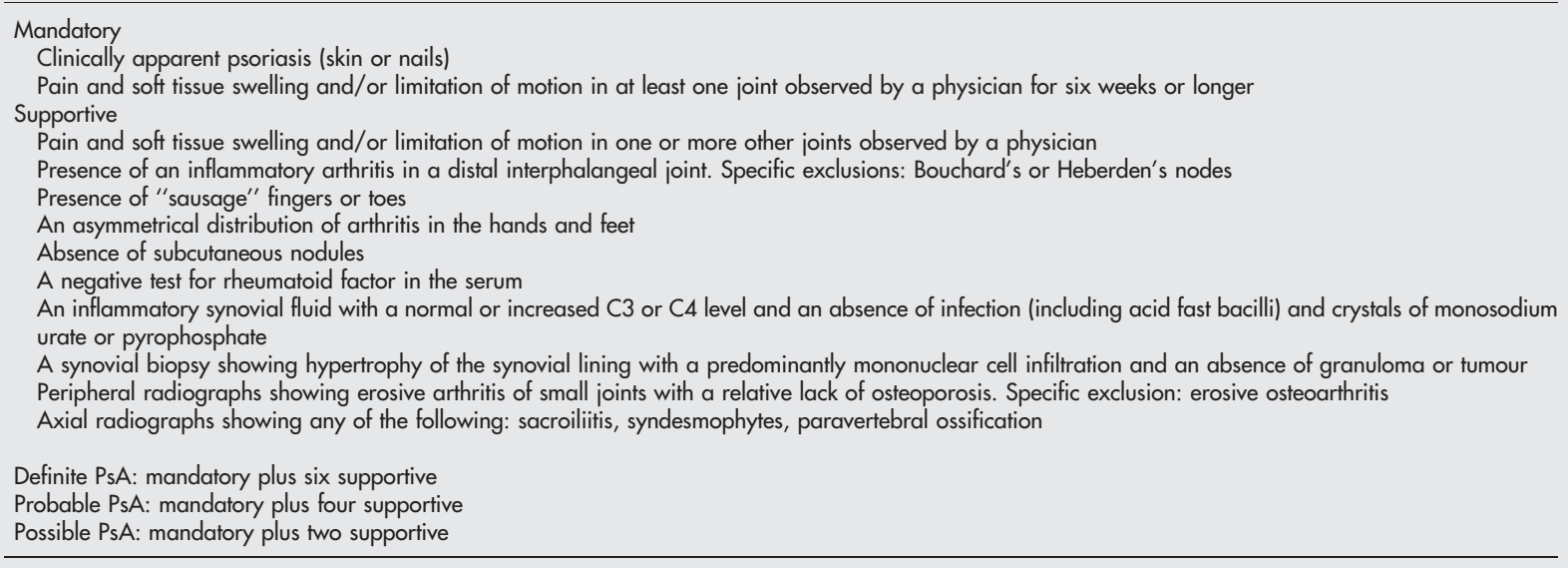


Table 2 Criteria for psoriatic arthritis proposed by Vasey and Espinoza ${ }^{32}$

Psoriatic arthritis is defined as criterion I plus one from either criterion II or III

Criterion I: Psoriatic skin or nail involvement

Criterion II: Peripheral pattern

(1) Pain and soft tissue swelling with or without limitation of movement of the distal interphalangeal joint for over four weeks

(2) Pain and soft tissue swelling with or without limitation of motion of the peripheral joints involved in an asymmetrical peripheral pattern for over four weeks. This includes a sausage digit

(3) Symmetrical peripheral arthritis for over four weeks, in the absence of rheumatoid factor or subcutaneous nodules

(4) Pencil in cup deformity, whittling of terminal phalanges, fluffy periostitis and bony ankylosis

Criterion III: Central pattern

(1) Spinal pain and stiffness with the restriction of motion present for over four weeks

(2) Grade 2 symmetric sacroiliitis according to the New York criteria

(3) Grade 3 or 4 unilateral sacroiliitis

Moll and Wright. ${ }^{14}$ These exclusions were: rheumatoid nodules, RA, crystal induced arthritis, grade IV osteoarthritis, Reiter's syndrome, and obvious inflammatory bowel disease.

In an attempt to make the diagnostic criteria for PsA more specific, Bennett, in 1979, proposed a new set of classification criteria. $^{31}$ Bennett's criteria combined the clinical features unique to PsA together with characteristic radiological features. In addition, two criteria, one based on synovial fluid analysis and the other based on synovial histology, were included. Despite the inclusion of radiographic, synovial fluid, and synovial biopsy items, it is still possible to diagnose PsA on clinical grounds alone (table 1). These criteria were not validated using patient data and have not been used in prospective studies in the complete format because of the difficulty of obtaining complete data. A number of other classification rules have been proposed but none has been validated on patient data. Vasey and Espinoza (table 2) simplified Bennett's rule recognising that there are two principal manifestations of PsA. ${ }^{32}$ Thus, only two criteria are required: psoriasis and one manifestation of either peripheral joint disease or spinal disease.

The ESSG derived classification criteria from consecutive patients with rheumatologist diagnosed spondyloarthropathy and controls with other rheumatic diseases. ${ }^{11}$ Although the intention of the criteria was for the diagnostic classification of the spondyloarthropathy group as a whole, particular types of spondyloarthropathy can be identified from the published classification criteria, including PsA (table 3). For the first time, it was recognised that PsA can actually occur in the absence of psoriasis.

More recently, McGonagle et al proposed a definition of PsA based on enthesopathy (table 4)..$^{33}$ There is a significant problem with evaluation of the original McGonagle criteria set because of the magnetic resonance imaging (MRI) requirements. It is unlikely that MRI would be practical for epidemiological research purposes. It is also likely that the MRI appearances in well established disease would show features of both enthesopathy and synovitis so that the

\begin{tabular}{l}
$\begin{array}{l}\text { Table } 3 \text { Modified ESSG criteria for psoriatic } \\
\text { arthritis }^{11}\end{array}$ \\
\hline Inflammatory spinal pain \\
or \\
Synovitis (either asymmetrical or predominantly lower limb) \\
and \\
One or more of the following: \\
Positive family history of psoriasis \\
Psoriasis \\
\hline
\end{tabular}

discriminant value of this feature would be markedly attenuated. In established disease, it has been suggested that plain radiographic evidence of enthesopathy might be more useful (D McGonagle, personal communication).

The only diagnostic rule specifically aimed at diagnosing PsA derived from patient data was developed by Fournié et al (table 5). ${ }^{34}$ It has yet to be tested in other populations. The items and weighting were selected using discriminant function and logistic regression analysis. The data were derived from a population of patients diagnosed by rheumatologists from a single clinic as having PsA, ankylosing spondylitis, and RA. A score of 11 points is required for the diagnosis of PsA (sensitivity 95\%, specificity 98\%, LR+ 47.5 in the original validation study) and, although the criteria include HLA data, it is possible to attain this threshold by clinical data alone. However, further validation would be required if the HLA criteria were omitted. It is also worth noting that these criteria permit the diagnosis of PsA in the absence of psoriasis or arthritis since a patient with HLA-B16/ 17, rheumatoid factor negative, and a family history of psoriasis scores 13 points.

In a recent study of 499 patients in Bradford and Milan, these existing criteria sets were tested against patient data collected from case notes and radiology review. ${ }^{35}$ Patients with RA $(n=156)$ or PsA $(n=343)$ were identified. Excluding the criteria of Fournié, which could not be applied in $24 \%$ of subjects, 446 cases could be classified by all of the other six methods. The most sensitive criteria were those of Vasey and Espinoza, McGonagle, and Gladman (99\%), whereas the others were significantly less sensitive (between $56 \%$ and $94 \%)$. The specificity of the rules was high and statistically similar (between 93\% and 99\%). The Fournié criteria were the most difficult to use, whereas the Vasey and Espinoza, and Moll and Wright criteria were the easiest (98\% of subjects were able to be classified).

Table 4 Modified McGonagle criteria for psoriatic arthritis $^{33}$

\begin{tabular}{l}
\hline Psoriasis or family history of psoriasis \\
Plus any one of: \\
Clinical inflammatory enthesitis \\
Radiographic enthesitis (replaces MRI evidence of enthesitis) \\
Distal interphalangeal joint disease \\
Sacroilitits/spinal inflammation \\
Uncommon arthropathies (SAPHO, spondylodiscitis, arthritis mutilans, \\
onchyo-pachydermo-periostitis, chronic multifocal recurrent \\
osteomyelitis) \\
Dactylitis \\
Monoarthritis \\
Oligoarthritis (four or less swollen joints) \\
\hline
\end{tabular}




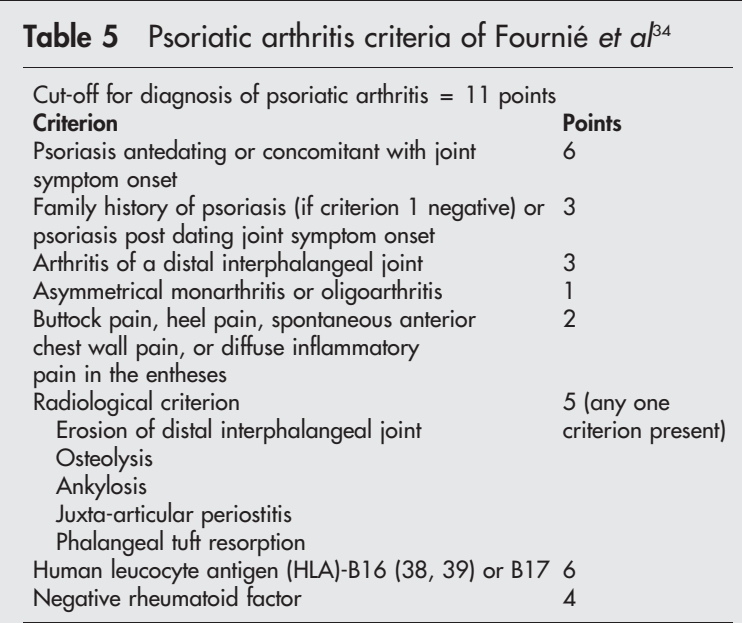

A two latent class model found test performance characteristics closely similar to those determined against a clinical diagnosis "gold standard". Logistic regression and classification and regression tree (CART) models suggested that negative rheumatoid factor was not necessary for diagnosis in the presence of other characteristic features of PsA. The presence of psoriasis differentiated between the two groups to such an extent that only this variable was selected as independently predictive when all variables were tested together. Even then, the data derived models failed to be more accurate than some of the criteria sets already proposed. However, it was possible to derive models that had reasonably good accuracy even without such key variables as psoriasis or rheumatoid factor.

\section{CASPAR STUDY GROUP}

The ClASsification of Psoriatic ARthritis (CASPAR) study group is an international group of investigators, all of whom have a record of research in PsA. The group has been collecting prospective clinical and radiological data on clinic patients since January 2002 with a target of 600 patients with PsA and 600 with a non-psoriatic arthropathy. Study completion was due at the end of July 2004 with results planned for 2005. A blood sample for HLA analysis and a serum sample for anti-CCP antibodies has been taken from each patient and stored. The project will test the diagnostic accuracy of existing classification rules and a seventh classification rule derived from CART analysis of a split sample. These results will identify the best clearly defined, properly validated classification rule for the purpose of case definition in the context of intervention trials for PsA. This study also proposes to address the question whether PsA is multiple coincident diseases or a single disease with multiple manifestations, by employing the statistical technique of latent class analysis. ${ }^{36}{ }^{37}$ This method will be able to test the hypothesis that the various diagnostic classification criteria are classifying patients into two classes (PsA and non-PsA), albeit with different degrees of accuracy. By testing the fit of the data to this hypothesised single dimensional disease, the unity of the disorder can be examined.

\section{SUMMARY AND CONCLUSIONS}

The study of PsA is difficult and has lagged behind the study of other arthropathies, particularly RA and ankylosing spondylitis. There has been some discrepancy in the literature regarding incidence and prevalence, with prevalence figures ranging from 20 to 100 per $100000 .^{38}$ One contributing reason for this discrepancy is that there are no universally agreed upon or properly validated case definitions of PsA. Since no validated case definitions exist, therapeutic studies could potentially include heterogeneous samples (people with and without the disorder of interest). By including patients who may have, for example, seronegative RA, the response rates of intervention trials may be positively biased towards therapies that affect the natural history of RA rather than PsA. Current classification criteria do not allow a clear separation between RA and PsA. ${ }^{39}$ As already noted, it is necessary to define combinations of more specific features that distinguish PsA from other arthropathies.

Establishing the case definition for PsA is therefore an important and fundamental issue. A number of published criteria exist, but we have no consensus on how best to define PsA. Robust clinical and basic science research into PsA requires the study of relatively homogeneous samples; this is not the case for current research. And finally, the most useful classification criteria should be validated in multiple centres, preferably in multiple countries, to avoid local diagnostic bias as the lack of a "gold standard" requires physician expert opinion to establish the diagnosis.

O'Neill and Silman ${ }^{40}$ state that the classification of PsA poses methodological difficulties: the spectrum of clinical disease is wide, the disease is relapsing and remitting, prevalence of disease is low, and methods for ascertaining past arthritis and psoriasis would need to be found. They note that existing classification criteria are theoretically derived rather than from patient data analysis. Further, three of the criteria sets are irrelevant to the clinical situation as they require either synovial fluid analysis/biopsy, HLA typing, or specialised imaging techniques. Most studies currently still use the original Moll and Wright definition, but it is now time to move on and develop new, internationally agreed upon criteria based on patient data. The CASPAR study should provide the way forward in the classification of PsA.

\section{Authors' affiliations}

P S Helliwell, Rheumatology and Rehabilitation Research Unit, University of Leeds, Leeds, UK

W J Taylor, Rehabilitation Teaching and Research Unit, Department of Medicine, Wellington School of Medicine and Health Sciences, University of Otago, Wellington, New Zealand

Correspondence to: P Helliwell, Rheumatology and Rehabilitation Research Unit, University of Leeds, 36, Clarendon Road, Leeds LS2 9NZ, UK; p.helliwell@leeds.ac.uk

\section{REFERENCES}

1 Wright V. Rheumatism and psoriasis; a re-evaluation. Am J Med 1959:27:454-62

2 Baker H, Golding DN, Thompson M. Psoriasis and arthritis. Ann Intern Med 1963;58:909-25.

3 Wright V. Psoriasis and arthritis. Ann Rheum Dis 1956;15:348-56.

4 Wright V. Psoriasis and arthritis: a comparative study of rheumatoid arthritis, psoriasis and arthritis associated with psoriasis. Arch Derm 1959;80:27-35.

5 Blumberg BS, Bunim JJ, Calkins E, Pirani CL, Zvaifler NJ. ARA nomenclature and classification of arthritis and rheumatism (tentative). Arthritis Rheum 1964;26:93-7.

6 Wright V, Moll JMH. Seronegative Polyarthritis. Amsterdam: North Holland Publishing Co, 1976.

7 Brewerton DA, Hart FD, Nicholls A, Caffrey M, James DC, Sturrock RD. Ankylosing spondylitis and HL-A 27. Lancet 1973;1:904-7.

8 Cats A. Is psoriatic arthritis an entity? In: Brooks PM, York JR, eds. Rheumatology. Amsterdam: Elsevier Science Publishers, 1985:295-301 9 Harrison BJ, Silman AJ, Barrett EM, Scott DG, Symmons DP. Presence of psoriasis does not influence the presentation or short-term outcome of patients with early inflammatory polyarthritis. J Rheumatol 1997;24:1744-9.

10 Arnett FC, Edworthy SM, Bloch DA, McShane DJ, Fries JF, Cooper NS, et al. The American Rheumatism Association 1987 revised criteria for the classification of rheumatoid arthritis. Arthritis Rheum 1988;31:315-24.

11 Dougados M, van der Linden S, Juhlin R, Huiffeldt B, Amor B, Calin A, et al. The European Spondylarthropathy Study Group preliminary criteria for the classification of spondylarthropathy. Arthritis Rheum 1991;34:1218-27.

12 Taylor WJ, Fellow DE, Helliwell PS. Case definition of psoriatic arthritis. Lancet 2000;356:2095. 
13 Moll JMH, Wright V. Psoriatic arthritis. Semin Arthritis Rheum 1973;3:55-78.

14 Gladman DD, Shuckett R, Russell ML, Thorne JC, Schachter RK. Psoriatic arthritis (PSA)-an analysis of 220 patients. Q J Med 1987:62:127-41.

15 Biondi Oriente C, Scarpa R, Pucino A, Oriente P. Psoriasis and psoriatic arthritis. Dermatological and rheumatological co-operative clinical report. Acta Derm Venereol Suppl (Stockh) 1989;146:69-71.

16 Helliwell P, Marchesoni A, Peters M, Barker M, Wright V. A re-evaluation of the osteoarticular manifestations of psoriasis. Br J Rheumatol $1991 ; 30: 339-45$.

17 Torre Alonso JC, Rodriguez Perez A, Arribas Castrillo JM, Ballina Garcia J, Riestra Noriega JL, Lopez Larrea C. Psoriatic arthritis (PA): a clinical, immunological and radiological study of 180 patients. $\mathrm{Br} J$ Rheumatol 1991;30:245-50

18 Jones SM, Armas JB, Cohen MG, Lovell CR, Evison G, McHugh NJ. Psoriatic arthritis: outcome of disease subsets and relationship of joint disease to nail and skin disease. Br J Rheumatol 1994;33:834-9.

19 Veale D, Rogers S, Fitzgerald O. Classification of clinical subsets in psoriatic arthritis. Br J Rheumatol 1994;33:133-8.

20 Jonsson T, Thorsteinsson J, Kolbeinsson A, Jonasdottir E, Sigfusson N, Valdimarsson $\mathrm{H}$. Population study of the importance of rheumatoid factor isotypes in adults. Ann Rheum Dis 1992;51:863-8.

21 Bizzaro N, Mazzanti G, Tonutti E, Villalta D, Tozzoli R. Diagnostic accuracy of the anti-citrulline antibody assay for rheumatoid arthritis. Clin Chem 2001;47:1089-93.

22 Helliwell PS, Hetthen J, Sokoll K, Green M, Marchesoni A, Lubrano E, et al. Joint symmetry in early and late rheumatoid and psoriatic arthritis: comparison with a mathematical model. Arthritis Rheum 2000;43:865-71.

23 Plunkett A, Marks R. A review of the epidemiology of psoriasis vulgaris in the community. Australas J Dermatol 1998;39:225-32.

24 Helliwell PS, Wright V. PsA: clinical features. In: Klippel JH, Dieppe PA, eds. Rheumatology. London: Mosby, 1998:6.21.1-6.21.8.

25 Gorter S, van der Heijde DM, van der Linden S, Houben H, Rethans JJ, Scherpbier AJ, et al. Psoriatic arthritis: performance of rheumatologists in daily practice. Ann Rheum Dis 2002;61:219-24.

26 Moll JM, Wright V. Familial occurrence of psoriatic arthritis. Ann Rheum Dis 1973;32:181-201.
27 McEwen C, Di Tatu D, Lingg C, Porini A, Good A, Rankin T. A comparative study of ankylosing spondylitis, and spondylitis accompanying ulcerative colitis, regional enteritis, psoriasis and Reiter's disease. Arthritis Rheum $1971 ; 14: 291-318$

28 Helliwell PS, Hickling P, Wright V. Do the radiological changes of classic ankylosing spondylitis differ from the changes found in the spondylitis associated with inflammatory bowel disease, psoriasis, and reactive arthritis? Ann Rheum Dis 1998;57:135-40.

29 de Vlam K, Mielants H, Verstaete KL, Veys EM. The zygapophyseal joint determines morphology of the enthesophyte. J Rheumatol 2000;27:1732-9.

30 Battistone MJ, Manaster BJ, Reda DJ, Clegg DO. The prevalence of sacroilitis in psoriatic arthritis: new perspectives from a large, multicenter cohort. A Department of Veterans Affairs Cooperative Study. Skeletal Radiol 1999;28:196-201.

31 Bennett RM. Psoriatic arthritis. In: McCarty DJ, ed. Arthritis and Related Conditions. Philadelphia: Lea \& Febiger, 1979:645.

32 Vasey FB, Espinoza LR. Psoriatic arthritis. In: Calin A, ed. Spondyloarthropathies. Orlando: Grune and Stratton, 1984:151-85.

33 McGonagle D, Conaghan PG, Emery P. Psoriatic arthritis: a unified concept twenty years on. Arthritis Rheum 1999;42:1080-6.

34 Fournie B, Crognier L, Arnaud C, Zabraniecki L, Lascaux-Lefebvre V, Marc V, et al. Proposed classification criteria of psoriatic arthritis. A preliminary study in 260 patients. Rev Rhum Engl Ed 1999;66:446-56.

35 Taylor WJ, Marchesoni A, Arreghini M, Sokol K, Helliwell PS. A comparison of the performance characteristics of classification criteria for the diagnosis of psoriatic arthritis. Semin Arthritis Rheum, 2005; (in press).

36 Faraone SV, Tsuang MT. Measuring diagnostic accuracy in the absence of a "gold standard". Am J Psychiatry 1994;151:650-7.

37 Yang I, Becker MP. Latent variable modeling of diagnostic accuracy. Biometrics 1997;3:948-58.

38 Veale DJ. The epidemiology of psoriatic arthritis: fact or fiction? J Rheumatol 2000;27:1 105-6.

39 Palazzi C, Olivieri I, Petricca A, et al. Rheumatoid arthritis or psoriatic symmetric polyarthritis? A difficult differential diagnosis. Clin Exp Rheumatol 2002;20:3-4.

$40 O^{\prime}$ Neill T, Silman AJ. Psoriatic arthritis. Historical background and epidemiology. Baillieres Clin Rheumatol 1994;8:245-61. 